\title{
Mal de débarquement syndrome diagnostic criteria: Consensus document of the Classification Committee of the Bárány Society
}

\author{
Yoon-Hee Cha ${ }^{\mathrm{a}, *}$, Robert W. Baloh ${ }^{\mathrm{b}}$, Catherine Cho ${ }^{\mathrm{c}}$, Måns Magnusson ${ }^{\mathrm{d}}$, Jae-Jin Songe, \\ Michael Strupp ${ }^{\mathrm{f}}$, Floris Wuyts ${ }^{\mathrm{g}}$ and Jeffrey P. Staab ${ }^{\mathrm{h}, 1}$ \\ ${ }^{a}$ Department of Neurology, University of Minnesota, Minneapolis, MN, USA \\ ${ }^{\mathrm{b}}$ Department of Neurology, University of California Los Angeles, Los Angeles, CA, USA \\ ${ }^{\mathrm{c}}$ Department of Neurology and Otolaryngology-Head and Neck Surgery, NYU Grossman School of Medicine, \\ $N Y, U S A$ \\ ${ }^{\mathrm{d}}$ Department of Otorhinolaryngology, Lund University, Lund, Sweden \\ ${ }^{\mathrm{e}}$ Department of Otorhinolaryngology Head-and-Neck Surgery, Seoul National University Bundang \\ Hospital, Seoul, South Korea \\ ${ }^{\mathrm{f}}$ Department of Neurology and German Center for Vertigo and Balance Disorders, Ludwig Maximilians \\ University, Munich, Germany \\ ${ }^{\mathrm{g}}$ Lab for Equilibrium Investigations and Aerospace (LEIA), University of Antwerp, Antwerp, Belgium \\ ${ }^{\mathrm{h}}$ Departments of Psychiatry and Psychology and Otorhinolaryngology-Head and Neck Surgery, \\ Mayo Clinic, Rochester, MN, USA
}

Received 22 February 2020

Accepted 1 July 2020

\begin{abstract}
We present diagnostic criteria for mal de débarquement syndrome (MdDS) for inclusion into the International Classification of Vestibular Disorders. The criteria include the following: 1] Non-spinning vertigo characterized by an oscillatory perception ('rocking,' 'bobbing,' or 'swaying') present continuously or for most of the day; 2] Onset occurs within 48 hours after the end of exposure to passive motion, 3] Symptoms temporarily reduce with exposure to passive motion (e.g. driving), and 4] Symptoms persist for $>48$ hours. MdDS may be designated as "in evolution," if symptoms are ongoing but the observation period has been less than 1 month; "transient," if symptoms resolve at or before 1 month and the observation period extends at least to the resolution point; or "persistent" if symptoms last for more than 1 month. Individuals with MdDS may develop co-existing symptoms of spatial disorientation, visual motion intolerance, fatigue, and exacerbation of headaches or anxiety. Features that distinguish MdDS from vestibular migraine, motion sickness, and persistent postural perceptual dizziness (PPPD) are reviewed. Motion-moderated oscillatory vertigo can also occur without a motion trigger, typically following another vestibular disorder, a medical illness, heightened psychological stress, or metabolic disturbance. Terminology for this non-motion triggered presentation has been varied as it has features of both MdDS and PPPD. Further research is needed into its phenomenological and biological relationship to MdDS, PPPD, and other vestibular disorders.
\end{abstract}

\footnotetext{
${ }^{1}$ Except for the first and last authors, authorship order is placed alphabetically.

${ }^{*}$ Corresponding author: Yoon-Hee Cha, Department of Neurology, University of Minnesota, Minneapolis, MN, USA. E-mail: ycha@umn.edu.
}

\section{Introduction}

As a multidisciplinary society of professionals dedicated to the advancement of vestibular science and clinical translation, the Bárány Society and 
the Classification Committee of the Bárány Society (CCBS) have commissioned diagnostic criteria for mal de débarquement syndrome (MdDS). The goal of creating an internationally sanctioned set of criteria for MdDS is to promote a common nomenclature for clinical diagnosis and to bring uniformity to research studies that investigate the pathophysiology of MdDS. These criteria will be included in the International Classification of Vestibular Disorders (ICVD) [1]. The relatively recent conceptualization that vestibular disorders can be due to functional alterations within the central nervous system without concurrent structural injury represents an important advancement within the field of neurotology [2]. This is particularly important to the future determination of the boundaries between MdDS and other functional vestibular disorders with which it shares common features.

\subsection{History}

Descriptions of individuals experiencing nonspinning vertigo, dizziness, and imbalance after sea-voyages have appeared in the medical literature for over 300 years [3]. This phenomenon was eventually termed 'mal de débarquement syndrome,' i.e., sickness of disembarkation. The earliest known description of MdDS in Western European literature appears to be from 1689 in the diaries of William III of Orange. The author describes how, after crossing the English Channel from the Netherlands to England, the soldiers experienced severe 'dissiness' described as, 'the very Ground seem'd to rowl up and down for some days, according to the manner of the Waves,' [3].

Erasmus Darwin (grandfather to Charles Darwin) included a description of MdDS under the "Vertigo' section of his medical tome Zoonomia in 1796 [4]. He described motion illusions occurring after sea voyages or stagecoach travel as, "undulating motion." Land-sickness, which may be a forme fruste of MdDS, was described by John Irwin in an 1881 Lancet article as a counterpoint to seasickness (aka mal de mer) [5].

\subsection{Terminology}

The description of MdDS in JT Reason and JJ Brand's 1975 classic work, "Motion Sickness," was an initial step towards bringing this disorder to the attention of the general medical practitioner [6].
Later, Brown and Baloh published the first case series of six individuals with MdDS in which they provided detailed descriptions of the clinical history and examination findings in these patients [7]. These descriptions formed the basis of the modern era of increased attention and interest in the clinical spectrum and underlying biology of MdDS.

\section{Methods}

In March 2017, the CCBS met in Berlin, Germany to introduce a format for developing criteria for MdDS. The selected chairperson (YHC) was approved to form a subcommittee of international specialists to begin developing criteria. The committee was formed and draft criteria were discussed in June 2018 in Uppsala, Sweden. Further communication occurred by email and phone calls between the group members and individually between the chair and subcommittee members. The draft proposal was presented to CCBS members in June 2019. The completed criteria were presented to the Bárány Society membership in January 2020. Further comments and suggestions were considered before submission for publication.

\section{Diagnostic criteria}

Though the term 'mal de débarquement,' has been previously applied to land-sickness lasting less than 48 hours, there are demographic and prognostic differences between the common short-term unsteadiness that happens immediately after landing and the syndrome that can last well beyond 48 hours [8-11]. The criteria set here thus recognize a distinction between non-pathological symptoms that last for less than 48 hours and those that last longer, which constitute the disorder of MdDS.

\subsection{Criteria for the diagnosis of mal de débarquement syndrome}

A. Non-spinning vertigo characterized by an oscillatory perception ('rocking,' 'bobbing,' or 'swaying') present continuously or for most of the day ${ }^{1}$

B. Onset occurs within 48 hours after the end of exposure to passive motion ${ }^{2}$ 
C. Symptoms temporarily reduce with exposure to passive motion ${ }^{3}$

D. Symptoms continue for $>48$ hours ${ }^{4}$

D.0 MdDS in evolution: symptoms are ongoing but the observation period has been less than 1 month

D.1 Transient MdDS: symptoms resolve at or before 1 month and the observation period extends at least to the resolution point

D.2 Persistent MdDS: symptoms last for more than 1 month

E. Symptoms not better accounted for by another disease or disorder.

\section{Notes}

1. Generally described as an oscillatory sensation such as 'rocking,' 'bobbing,' or 'swaying.' The term 'oscillatory' is used here to describe the subjective perception of the motion and not the temporal meaning of vertigo that comes and goes. We further define 'rocking' as a fore-andaft perception, 'bobbing' as an up-and-down perception, and 'swaying' as a side-to-side perception. Perceptions can be mixed and can also change direction with time. There can be superimposed feelings of gravitational force on the body in any translational direction.

2. Typical triggers include transportation vessels such as boats, airplanes, automobiles, and trains but can also include swaying buildings, waterbeds, exercise equipment and other platforms that passively move the individual $[7,12$, 13]. There may be sequential exposure to more than one trigger, e.g. water travel followed by air travel. The key features of the triggers are an oscillatory or periodic stimulus coupled with some minimal duration of exposure, generally on the order of hours. The travel experience that leads to an episode of MdDS does not appear to have any particular features in terms of related illness or motion sickness but it is common for individuals with MdDS to have experienced a concurrent physical or psychological stressor during the travel that triggered their symptoms [13, 14]. Hormonal influences such as the peri-menopausal or peri-menstrual state at the time of exposure may also be a risk factor [15].

There has yet to be a clear report of persistent oscillatory vertigo triggered by exposure to virtual reality. Prior reports of MdDS being caused by virtual reality stimuli referenced a study that reported less than 25 minutes of dizziness (not vertigo) occurring after a virtual reality game [8, 16-18]. This would not meet criteria for MdDS set here, nor would it meet a threshold for clinical relevance.

Unsteadiness and orthostatic intolerance have been described following space travel but persistent oscillatory vertigo is not a typical post-space travel symptom [19-21].

3. Driving in an automobile or returning to the triggering stimulus such as getting back on the boat will temporarily relieve the vertigo of MdDS. Symptoms frequently rebound, however when the stimulus stops, e.g. automobile stops at a traffic light. Walking may also relieve the symptoms temporarily but the effect is variable depending on the pace and the perceived underlying rhythm. In some individuals, lying down increases the motion perception [13].

4. Short duration symptoms lasting less than 48 hours are extremely common even among healthy young individuals [8-11]. Persistent symptoms that make up the clinically relevant syndrome of MdDS are significantly less common and can lead to high levels of morbidity $[13,22]$. If a patient presents with symptoms less than one month in duration and one month of observation time has not yet passed, they should be diagnosed as D0, MdDS 'in evolution.' "Transient MdDS," (D1) can only be diagnosed retrospectively if symptoms remit in less than one month. Persistent MdDS (D2) is diagnosed once symptoms persist beyond one month.

\section{Comment}

\subsection{Harmonization with ICVD classification}

According to ICVD, which established a nonhierarchical distinction between the terms "dizziness," and "vertigo," and further distinguished 'spinning' from 'non-spinning' vertigo, the perception of motion described in MdDS would be classified as 'non-spinning vertigo.' Under ICVD classification, the perception of oscillating motion experienced in MdDS would generally be considered a form of 'internal vertigo,' defined as the "sensation of self-motion when no self-motion is occurring or the sensation of distorted self-motion during an other- 
wise normal head movement," [1]. MdDS would be coded as 1.2.7 "Other triggered vertigo," specifically, vertigo induced "after prolonged exposure to passive motion (as occurs following sea voyages)."

The counterpart to 'internal vertigo,' is 'external vertigo,' currently defined as "the false sensation that the visual surround is spinning or flowing" [1]. This is not a core symptom of MdDS. Though visual perceptions of either oscillatory motion or vibration can occasionally be noted in MdDS, if visual motion illusions of any form are the predominant symptoms, an alternate diagnosis to MdDS should be sought.

\subsection{Additional features}

A dimension of MdDS that is not captured in current ICVD terminology relates to the haptic or somatic perception of the environment in motion, e.g. 'the ground undulating,' or 'rocking on a boat.' This is perceived as forces acting upon the head or body rather than as visual phenomena and would thus not be consistent with the ICVD definition of external vertigo, which is defined as a visual phenomenon. The strength of this force on the body can fluctuate as the reference point of the motion source shifts between the individual and the environment. The basis for this percept in MdDS is currently unknown.

Individuals with MdDS may concurrently experience dizziness, defined as, "a sensation of disturbed or impaired spatial orientation without a false or distorted sense of motion." The dizziness may be spontaneous (2.1) or may have one or more triggers (2.2) including "after prolonged exposure to passive motion (as occurs following sea voyages)," [1]. Both head-motion dizziness (2.2.2) and visually-induced dizziness (2.2.3) are particularly common in MdDS and may occur concurrently with core MdDS symptoms [23].

Other symptoms that may be part of the MdDS spectrum include cognitive slowing, fatigue, photophobia, phonophobia, headache, and anxiety [23]. These occur to varying degrees in each individual and their presence does not add diagnostic accuracy given their non-specific nature.

\section{MdDS clinical features}

\subsection{Epidemiology}

One tertiary level neurotology practice reported that $1.3 \%$ of their clinic patients were diagnosed with
MdDS compared to $8.6 \%$ with Ménière's disease over a five-year period [24]. It should be kept in mind that MdDS is frequently misdiagnosed as other disorders in the general medical community (see Section 6), however, which makes a direct population measure difficult [25].

\subsection{Demographics}

The temporary experience of post-motion exposure non-spinning vertigo lasting less than 48 hours is common in otherwise healthy individuals and shows a roughly equal sex distribution $[11,26]$. However, MdDS lasting more than 48 hours and particularly lasting more than 1 month is overwhelmingly represented by women $(75-100 \%)[13,27,28]$. The age of onset peaks between 40-49 years and follows a normal distribution [13]. MdDS has been reported in children as young as 12 years old and in adults well into their 70's, though unpublished clinical experience within the present committee indicates that the upper and lower limits do extend further [13].

\subsection{Temporal course}

An individual may experience more than one lifetime episode of MdDS as well as episodes of similar but non-motion triggered persistent oscillating vertigo that follows or may be interleaved with motion-triggered episodes [23]. Subsequent episodes are usually, but not always, longer than prior episodes with the chance of a spontaneous recovery declining as an exponential function of duration of illness [23].

MdDS symptom severity within a current episode may fluctuate due to factors such as stress, sleep deprivation, and hormonal factors, with the latter being represented by worsened peri-menstrual symptoms $[13,15]$. Vertigo intensity may be modulated by body position. Though standing is generally worse than sitting or lying flat, about a third of individuals report increased vertigo amplitude with lying down [13]. Thus, unlike disorders that are primarily characterized by unsteadiness on being upright, the vertigo of MdDS persists in different body positions.

\subsection{Common co-occurring symptoms}

Individuals with MdDS commonly have additional symptoms that develop with or subsequent to the onset of their disorder. These include sensations of spatial disorientation, fatigue, visual motion intolerance, headaches, and anxiety $[13,23]$. These may 
predate the onset of MdDS and may worsen with the onset of MdDS but are not considered core elements of MdDS. Their potential pathophysiological connections to MdDS are unknown. When any of these symptoms are severe enough to constitute a diagnosable condition of their own (e.g., a specific headache or anxiety disorder), these diagnoses should be made in addition to MdDS and treated with established therapies.

\section{Laboratory examinations}

The diagnosis of MdDS is based on clinical history alone, relevant to Criteria A-D. There are no findings on physical examination, laboratory testing, or imaging that are pathognomonic of MdDS [23, 29]. Direction changing static positional nystagmus in the dark has been noted in MdDS but is a non-specific finding that is also seen in people without MdDS [7].

When there is a typical onset of symptoms according to the criteria set forth, the yield of vestibular and auditory function testing is very low and may even derail an accurate diagnosis by uncovering nonspecific or false positive findings [23, 28]. Similarly, available literature indicates that clinically available structural brain imaging with MRI or CT is of low yield in the diagnosis of MdDS [23]. Supplementary testing of semicircular canal, otolith, or cochlear function and/or brain imaging may be performed in cases with atypical features such as those with concurrent hearing loss, ocular motor abnormalities, or neurological deficits [24].

Alterations in vestibulo-ocular reflexes and changes in brain metabolism and functional connectivity have been identified in neuroimaging research in MdDS [30-32]. However, these neuroimaging technologies are not recommended for clinical diagnoses.

\section{Differential diagnosis}

\subsection{Vestibular migraine}

Vestibular migraine is currently defined as an episodic disorder in which at least five distinct episodes of vestibular symptoms lasting from 5 minutes to 72 hours occur in an individual with a history of typical migraine headache and in which $50 \%$ of the vertigo episodes are associated with one or more non-vertigo migraine features, e.g. aura, pho- tophobia and phonophobia [33]. Episodic vestibular symptoms related to migraine may respond to conventional migraine therapy [34-41].

Vestibular migraine and MdDS do share many features. Both occur more commonly in women, are worsened during the peri-menstrual period, and involve vestibular symptoms that start later in life $[13,33,42]$. Migraine headaches can develop concurrently with the onset of MdDS and worsen with the development of MdDS [43]. Individuals who develop non-motion triggered persistent vertigo that is phenotypically similar to MdDS (see Section 7) have a higher rate of pre-existing migraine headaches than those who develop MdDS as defined in these criteria, indicating that there may be some shared biology between vestibular migraine and persistent perceptions of oscillation [23, 43].

Despite the shared clinical features, there are a number of differences between vestibular migraine and MdDS. The percentage of individuals with a history of migraine headaches prior to the onset of MdDS does not appear to be higher than population baseline [23, 25]. Though migraine headaches can develop with the onset of MdDS, over $50 \%$ of MdDS sufferers do not meet criteria for migraine at any point in their lives [43]. Headache associated with MdDS may also be described as a persistent pressure rather than as a distinct unilateral throbbing pain typical of migraine [43]. Finally, MdDS and non-motion triggered persistent oscillating vertigo disorders present with a chronic course that would not meet the temporal restrictions of the criteria for vestibular migraine [13].

Due to the distinctions between MdDS and vestibular migraine, a diagnosis of vestibular migraine should be made in addition to MdDS if criteria for both disorders are met, separately. Further investigations are needed to determine how much biological overlap there is between these disorders.

\subsection{Motion sickness}

Passive or active head motion or motion of the visual field may induce motion sickness. Motion sickness includes a variable combination of nausea, stomach awareness, sweating, drowsiness, headache, or eye strain/blurred vision (visually-induced motion sickness) $[6,44]$. It is not associated with vertigo as defined by ICVD [1]. Motion sickness occurs during the motion stimulus and builds gradually with longer exposure. Though some symptoms may persist beyond the stimulus exposure, they do not start 
de novo after the stimulus has completely ended. This feature is unlike MdDS, which starts after the motion stimulus has ended.

Individuals with MdDS may experience motion sickness during the exposure that triggered their symptoms and may also experience nausea early in their symptom course [14]. However, nausea generally decreases with time in MdDS and is not a hallmark feature [23]. Moreover, the nulling of oscillating vertigo with driving or riding in a car would imply that MdDS is pathophysiologically distinct from motion sickness.

\subsection{Persistent postural perceptual dizziness}

Persistent Postural Perceptual Dizziness (PPPD) is defined as a chronic vestibular disorder lasting for at least three months that manifests with dizziness, unsteadiness, or non-spinning vertigo [45]. Upright posture, active or passive motion, and visual stimulation exacerbate PPPD, though symptoms of PPPD may fluctuate with or without specific provocation [45]. Persistent Postural Perceptual Dizziness is triggered by vestibular or neurologic disorders, psychological distress, or medical illnesses. Thus, the precipitants and provocations of MdDS differ from those of PPPD. MdDS is triggered by exposure to passive motion, whereas PPPD is triggered by events that disrupt balance function. Individuals with MdDS, as a defining criterion for the disorder, experience a temporary reduction in symptoms with re-exposure to passive motion, whereas individuals with PPPD are much more likely to experience an increase in symptoms as a defining characteristic. Furthermore, individuals with PPPD experience an exacerbation of symptoms with exposure to complex visual motion stimuli as a defining characteristic of the disorder. Sensitivity to visual motion stimuli occurs in most individuals with MdDS but is not one of its core features [23].

\section{Areas of uncertainty}

A phenotypically similar disorder to MdDS has been described both in clinical reports and in interventional studies in which persistent vertigo characterized by a perception of oscillation (rocking, bobbing, swaying) occurs without a prior motion trigger. This syndrome has been variably called 'spontaneous MdDS,' 'aberrant MdDS,' 'atypical MdDS,' 'MdDS-like,' 'non motion-triggered MdDS,' and 'mixed-MdDS.' [13, 31, 43, 46]. The latter term has been used to describe cases in which a nonmotion triggered episode follows a motion-triggered episode, recognizing that individuals may experience both motion-triggered and non-motion triggered episodes [23].

The issue of how to categorize the non-motion triggered cases of persistent oscillating vertigo with respect to MdDS and PPPD was intensely examined by committee members. There was support for aligning the non-motion triggered presentation with each, both, and neither based on similar and distinct clinical features of each diagnostic group (Table).

The main point of discussion in the committee was the effect of active or passive motion on symptoms. These currently non-categorized individuals would not meet Criterion B but would meet Criteria $\mathrm{A}, \mathrm{C}$, and $\mathrm{D}$ for MdDS. Inciting factors for this non-motion triggered group align closely with those for PPPD, however, Criterion B for PPPD requires that symptoms be exacerbated by active or passive motion without regard to direction. It is further clarified in the Comments section of PPPD criteria that this motion can be self-generated or involve passive motion such as riding in a vehicle. Thus, the non-motion triggered motion-moderated oscillating vertigo group would be excluded from PPPD. Adding a layer of nuance, however, is that individuals with PPPD may prefer small amounts of motion such as walking or riding a bicycle over standing completely still [45].

Theories of the underlying mechanisms of MdDS, PPPD, and the non-motion triggered group were raised, but ultimately it was decided that there are currently not enough data to support the inclusion of the non-motion triggered motion moderated oscillating vertigo group into either category.

\section{Future directions}

The committee was in unanimous agreement that further scientific investigations were required to accurately align biological foundations of MdDS with ICVD nomenclature and to more clearly identify its distinctions from phenotypically related disorders. The ICVD currently includes some disorders defined on phenomenological descriptions (vestibular migraine) [33], others with diagnostic criteria that include both symptoms and associated signs (BPPV, Ménière's disease, bilateral vestibulopathy, presbyvestibulopathy, hemodynamic orthostatic 
Table 1

Comparison of clinical features of MdDS, PPPD, and an overlap syndrome

\begin{tabular}{|c|c|c|c|}
\hline & $\begin{array}{l}\text { Mal de Debarquement } \\
\text { Syndrome (MdDS) }\end{array}$ & $\begin{array}{l}\text { Non motion-triggered } \\
\text { motion-moderated } \\
\text { oscillating vertigo }\end{array}$ & $\begin{array}{c}\text { Persistent Postural-Perceptual } \\
\text { Dizziness (PPPD) }\end{array}$ \\
\hline \multicolumn{4}{|l|}{ Core symptoms } \\
\hline Non-spinning vertigo & $\begin{array}{l}\text { Internal, rarely external } \\
\text { oscillatory }\end{array}$ & $\begin{array}{c}\text { Internal, rarely external } \\
\text { oscillatory }\end{array}$ & $\begin{array}{l}\text { Internal or external, may be } \\
\text { oscillatory at times }\end{array}$ \\
\hline Unsteadiness & May occur & May occur & Defining feature \\
\hline $\begin{array}{r}\text { Hypersensitivity to complex visual } \\
\text { motion stimuli }\end{array}$ & May occur & May occur & Defining feature \\
\hline \multicolumn{4}{|l|}{ Precipitants } \\
\hline Motion (boats, planes, cars, etc.) & Yes & No & No \\
\hline $\begin{array}{r}\text { Other medical, psychologic, } \\
\text { or vestibular events }\end{array}$ & No & Yes & Yes \\
\hline \multicolumn{4}{|l|}{ Response to provocation } \\
\hline Reduced by passive motion & Yes & Yes & No \\
\hline Worsened by passive motion & No & Rare & Yes \\
\hline Worsened by active motion & Variable & Variable & Yes \\
\hline Worsened by upright posture & Yes & Yes & Yes \\
\hline \multicolumn{4}{|l|}{ Diagnostic testing } \\
\hline Vestibular function testing & Normal & Normal & $\begin{array}{l}\text { Variable (related to triggering } \\
\text { condition) }\end{array}$ \\
\hline Clinical neuroimaging & Normal & Normal & Normal \\
\hline \multicolumn{4}{|l|}{ Treatment response } \\
\hline SSRI/SNRI & Yes & Yes & Yes \\
\hline Benzodiazepine & Yes & Yes & Yes (not first line) \\
\hline Physical therapy & No & No & Yes \\
\hline Psychotherapy & No & Unknown & Yes \\
\hline \multicolumn{4}{|l|}{ Findings from neuroimaging research } \\
\hline fMRI & $\begin{array}{l}\text { Long-range cortical, insular, } \\
\text { limbic, and cerebellar } \\
\text { connectivity changes }\end{array}$ & Unknown & $\begin{array}{l}\text { Long-range cortical, } \\
\text { peri-opercular, limbic, and } \\
\text { cerebellar connectivity } \\
\text { changes }\end{array}$ \\
\hline PET & $\begin{array}{l}\text { Hypermetabolism in the } \\
\text { entorhinal cortex \& } \\
\text { amygdala; Hypometabolism } \\
\text { in prefrontal \& temporal } \\
\text { cortices }\end{array}$ & Unknown & Unknown \\
\hline
\end{tabular}

dizziness/vertigo) [47-51], and one that has a criterion based on response to treatment (sodium channel blockers in vestibular paroxysmia) [52]. Broadening of the spectrum of vestibular disorders may require adapting new terminology in the future to avoid any inherent inconsistencies. For example, if the experience of persistent oscillating vertigo is distinct from other forms of non-spinning vertigo in terms of demographics, associated symptoms, triggers, modulating factors, and future health implications, it may be justified to create its own designation.

An area of uncertainty with regards to MdDS is whether improvement with passive motion is a critical feature that differentiates persistent vertigo characterized by oscillatory perceptions from other vestibular disorders, regardless of cause. There is evidence for prognostic significance of the mechanistic pathway taken to symptom development. For example, delayed endolymphatic hydrops due to prior inner ear damage has a worse prognosis than idiopathic Ménière's disease though both lead to episodes of vertigo and hearing loss [53, 54]. Similarly, persistent oscillating vertigo that develops after a non-motion trigger has a lower treatment response rate than motion triggered MdDS [13, 43, 46].

Some areas of future exploration are proposed to resolve this ambiguity:

Qualitative studies: Distinct elements between these groups (motion and non-motion triggered) may be too subtle to capture with currently available quantitative methods. There may be benefits to evaluating differences in each group based on themes such as natural history and future health implications.

Gait analysis: Gait strategies activated by individuals with MdDS vs PPPD vs other disorders of persistent vertigo might be differentiated. For exam- 
ple, walking speed may be affected by the periodicity of the internal vertigo and whether this perception is nulled by motion.

Postural testing: Differences in postural strategies engaged during balance perturbation in each group may be assessed, especially those triggered by visual stimuli.

Associated syndromes: The temporal course and impact of migraine headache, psychiatric disorders, cognitive domains, or family history may reveal dissociable features.

Treatment response: Response to specific classes of medication, physical therapy, vestibular therapy, psychotherapy, or neuromodulation may show different trends in each group.

Imaging studies: Resting state and task induced functional connectivity as well as morphological measurements may show correlations between visual, vestibular, insular, limbic, prefrontal, and cerebellar regions that may provide insights into the operations of central vestibular connections that undergird the perception of head and body stability in each group.

\section{Acknowledgments}

The working meetings for the International Classification of Vestibular Disorders (ICVD) are financially supported by the International Bárány Society and by Neuro+e.V., Berlin, Germany, a nonprofit association for neurological research.

\section{References}

[1] A. Bisdorff, M. Von Brevern, T. Lempert and D.E. NewmanToker, Classification of vestibular symptoms: towards an international classification of vestibular disorders, $J$ Vestib Res 19 (2009), 1-13.

[2] J.P. Staab, Functional and psychiatric vestibular disorders, Handb Clin Neurol 137 (2016), 341-351.

[3] J. Whittie, An exact diary of the late expedition of His Illustrious Highness the Prince of Orange, 1689, Bradford on Avon, UK: Ex Libris Press (1986).

[4] E. Darwin, Zoonomia, or The Laws of Organic Life, Volume I : Second Edition. Printed for J. Johnson, St. Paul's ChurchYard, London, (1796).

[5] J.A. Irwin, The pathology of sea-sickness, The Lancet 118 (1881), 907-909.

[6] J.T. Reason and J.J. Brand, Motion Sickness, London, New York, San Francisco: Academic Press, (1975).

[7] J.J. Brown and R.W. Baloh, Persistent mal de debarquement syndrome: a motion-induced subjective disorder of balance, Am J Otolaryngol 8 (1987), 219-222.
[8] Z. Nachum, A. Shupak, V. Letichevsky, J. Ben-David, D. Tal, A. Tamir, Y. Talmon, C.R. Gordon and M. Luntz, Mal de debarquement and posture: reduced reliance on vestibular and visual cues, Laryngoscope 114 (2004), 581-586.

[9] D. Tal, G. Wiener and A. Shupak, Mal de debarquement, motion sickness and the effect of an artificial horizon, $J$ Vestib Res 24 (2014), 17-23.

[10] C.R. Gordon, A. Shupak, Z. Nachum and T.C. Hain, Mal de debarquement, Arch Otolaryngol Head Neck Surg 126 (2000), 805-806

[11] H. Cohen, Mild mal de debarquement after sailing, Ann $N$ Y Acad Sci 781 (1996), 598-600.

[12] I.W.S. Mair, The mal de debarquement syndrome, J Audiol Med 5 (1996), 21-26.

[13] Y.H. Cha, Y.Y. Cui and R.W. Baloh, Comprehensive Clinical Profile of Mal De Debarquement Syndrome, Front Neurol 9 (2018), 261.

[14] T.C. Hain, P.A. Hanna and M.A. Rheinberger, Mal de debarquement, Arch Otolaryngol Head Neck Surg 125 (1999), 615-620.

[15] V. Mucci, J.M. Canceri, R. Brown, M. Dai, S.B. Yakushin, S. Watson, A. Van Ombergen, Y. Jacquemyn, P. Fahey, et al. Mal de Debarquement Syndrome: A Retrospective Online Questionnaire on the Influences of Gonadal Hormones in Relation to Onset and Symptom Fluctuation, Front Neurol 9 (2018), 362.

[16] A. Van Ombergen, V. Van Rompaey, L.K. Maes, P.H. Van de Heyning and F.L. Wuyts, Mal de debarquement syndrome: a systematic review, J Neurol 263 (2016), 843-854.

[17] V. Nwagwu, R. Patel and J. Okudo, Mal de Debarquement Syndrome: A Rare Entity-A Case Report and Review of the Literature, Case Rep Otolaryngol 2015, 918475.

[18] S.V. Cobb and S.C. Nichols, Static posture tests for the assessment of postural instability after virtual environment use, Brain Res Bull 47 (1998), 459-464.

[19] E. Hallgren, L. Kornilova, E. Fransen, D. Glukhikh, S.T. Moore, G. Clément, A. Van Ombergen, H. MacDougall, I. Naumov and F.L. Wuyts, Decreased otolithmediated vestibular response in 25 astronauts induced by long-duration spaceflight, J Neurophysiol 115 (2016), 3045-3051.

[20] F.O. Black and W.H. Paloski, Computerized dynamic posturography: What have we learned from space? Otolaryngol Head Neck Surg 118 (1998), S45-S51.

[21] K. Bacal, R. Billica and S. Bishop, Neurovestibular symptoms following space flight, J Vestib Res 13 (2003), 93-102.

[22] A. Macke, A. Leporte and B.C. Clark, Social, societal, and economic burden of mal de debarquement syndrome, $\mathrm{J} \mathrm{Neu}$ rol 259 (2012), 1326-1330.

[23] Y.H. Cha, J. Brodsky, G. Ishiyama, C. Sabatti and R.W. Baloh, Clinical features and associated syndromes of mal de debarquement, J Neurol 255 (2008), 1038-1044.

[24] Y.H. Cha, Less common neuro-otologic disorders, Continuum (Minneap Minn) 18 (2012), 1142-1157.

[25] V. Mucci, J.M. Canceri, R. Brown, M. Dai, S. Yakushin, S. Watson, A. Van Ombergen, V. Topsakal, P.H. Van de Heyning, P.H., et al. Mal de Debarquement Syndrome: a survey on subtypes, misdiagnoses, onset and associated psychological features, J Neurol 265 (2018), 486-499.

[26] T.A. Stoffregen, F.C. Chen, M. Varlet, C. Alcantara and B.G. Bardy, Getting Your Sea Legs, PLoS One 8 (2013), e66949.

[27] T.C. Hain and M. Cherchi, Mal de débarquement syndrome, Handb Clin Neurol 137 (2016), 391-395.

[28] Y.H. Cha, Mal de debarquement, Semin Neurol 29 (2009), $520-527$. 
[29] Y.H. Cha and R.W. Baloh, Migraine associated vertigo, $J$ Clin Neurol 3 (2007), 121-126.

[30] Y.H. Cha, S. Chakrapani, A. Craig and R.W. Baloh, Metabolic and functional connectivity changes in mal de debarquement syndrome, PLoS One 7 (2012), e49560.

[31] M. Dai, B. Cohen, E. Smouha and C. Cho, Readaptation of the vestibulo-ocular reflex relieves the mal de debarquement syndrome, Front Neurol 5 (2014), 1-6.

[32] H. Yuan, G. Shou, D. Urbano, L. Ding and Y.H. Cha, Resting state functional connectivity signature of treatment effects of rTMS in Mal de Debarquement Syndrome, Brain Connect 7 (2017), 617-626.

[33] T. Lempert, J. Olesen, J. Furman, J. Waterston, B. Seemungal, J. Carey, A. Bisdorff, M. Versino, S. Evers and D. Newman-Toker, Vestibular migraine: Diagnostic criteria, $J$ Vestib Res 22 (2012), 167-172.

[34] G.D. Johnson, Medical management of migraine-related dizziness and vertigo, Laryngoscope 108 (1998), 1-28.

[35] J.M. Furman, D.A. Marcus and C.D. Balaban, Rizatriptan reduces vestibular-induced motion sickness in migraineurs, $J$ Headache Pain 12 (2011), 81-88.

[36] M. Salviz, T. Yuce, H. Acar, A. Karatas and R.M. Acikalin, Propranolol and venlafaxine for vestibular migraine prophylaxis: A randomized controlled trial, Laryngoscope 126 (2016), 169-174.

[37] S.P. Cass, J.M. Furman, K. Ankerstjerne, C. Balaban, S. Yetiser and B. Aydogan, Migraine-related vestibulopathy, Ann Otol Rhinol Laryngol 106 (1997), 182-189.

[38] J.M. Furman, C.D. Balaban, R.G. Jacob and D.A. Marcus, Migraine-anxiety related dizziness (MARD): a new disorder? J Neurol Neurosurg Psychiatry 76 (2005), 1-8.

[39] A. Radtke, M. von Brevern, H. Neuhauser, T. Hottenrott and T. Lempert, Vestibular migraine: long-term follow-up of clinical symptoms and vestibulo-cochlear findings, $\mathrm{Neu}$ rology 79 (2012), 1607-1614.

[40] H.K. Neuhauser, A. Radtke, M. von Brevern, M. Feldmann, F. Lezius, T. Ziese and T. Lempert, Migrainous vertigo: prevalence and impact on quality of life, Neurology 67 (2006), 1028-1033.

[41] H.K. Neuhauser, M. von Brevern, A. Radtke, F. Lezius, M. Feldmann, T. Ziese and T. Lempert, Epidemiology of vestibular vertigo: a neurotologic survey of the general population, Neurology 65 (2005), 898-904.

[42] Y.H. Cha, H. Lee, L.S. Santell and R.W. Baloh, Association of benign recurrent vertigo and migraine in 208 patients, Cephalalgia 29 (2009), 550-555.

[43] Y.H. Cha and Y. Cui, Rocking dizziness and headache: a two-way street. Cephalalgia 33 (2013), 1160-1169.
[44] J.F. Golding, Motion sickness. Handb Clin Neurol 137 (2016), 371-390.

[45] J.P. Staab, A. Eckhardt-Henn, A. Horii, R. Jacob, M. Strupp, T. Brandt and A. Bronstein, Diagnostic Criteria for Persistent Postural-Perceptual Dizziness (PPPD): Consensus document of the Committee for the Classification of Vestibular Disorders of the Bárány Society, J Vest Res 27 (2017), 191-208.

[46] M. Dai, B. Cohen, C. Cho, S. Shin and S.B. Yakushin, Treatment of the Mal de Debarquement Syndrome: A 1-Year Follow-up, Front Neurol 8 (2017), 175.

[47] M. von Brevern, P. Bertholon, T. Brandt, T. Fife, T. Imai, D. Nuti and D.E. Newman-Toker, Benign paroxysmal positional vertigo: Diagnostic criteria Consensus document of the Committee for the Classification of Vestibular Disorders of the Bárány Society, J Vest Res 25 (2015), 105-117.

[48] J.A. Lopez-Escamez, J. Carey, W.H. Chung, J.A. Goebel, M. Magnusson, M. Mandalà, D.E. Newman-Toker, M. Strupp, M. Suzuki, et al. Diagnostic criteria for Menière's disease, J Vestib Res 25 (2015), 1-7.

[49] M. Strupp, J.S. Kim, T. Murofushi, D. Straumann, J.C. Jen, S.M. Rosengren, C.C. Della Santina and H. Kingma, Bilateral Vestibulopathy: Diagnostic criteria. Consensus document of the Classification Committee of the Bárány Society, J Vestib Res 27 (2017), 177-189.

[50] Y. Agrawal, R. Van de Berg, F. Wuyts, L. Walther, M. Magnusson, E. Oh, M. Sharpe and M. Strupp, Presbyvestibulopathy: Diagnostic criteria, Consensus document of the classification committee of the Bárány Society, $J$ Vestib Res 29 (2019), 161-170.

[51] H.A. Kim, A. Bisdorff, A.M. Bronstein, T. Lempert, M. Rossi-Izquierdo, J.P. Staab, M. Strupp and J.S. Kim, Hemodynamic orthostatic dizziness/vertigo: Diagnostic criteria. Consensus document of the Committee for the Classification of Vestibular Disorders of the Bárány Society, $J$ Vestib Res 29 (2019), 45-56.

[52] M. Strupp, J.A. Lopez-Escamez, J.S. Kim, D. Straumann, J.C. Jen, J. Carey, A. Bisdorff and T. Brandt, Vestibular paroxysmia: Diagnostic criteria, J Vestib Res 26 (2016), 409-415.

[53] U. Friberg, J. Stahle and A. Svedberg, The natural course of Meniere's disease, Acta Oto-Laryngologica 96 (1983), 72-77.

[54] T. Kamei, Delayed endolymphatic hydrops as a clinical entity, Int Tinnitus J 10 (2004), 137-143. 